\title{
RESULTS ON POSITIVE SOLUTIONS OF ELLIPTIC EQUATIONS WITH A CRITICAL HARDY-SOBOLEV OPERATOR*
}

\author{
DAOMIN $\mathrm{CAO}^{\dagger}$ AND YANYAN $\mathrm{LI}^{\ddagger}$ \\ Dedicated to Neil Trudinger with respect and friendship \\ on the occasion of his sixty fifth birthday
}

Key words. Hardy-Sobolev operator, uniqueness, entire solutions.

AMS subject classifications. 35J20, 35J65

1. Introduction. Let $N \geq 3,1 \leq k<N$ and $\mathbb{R}^{N}=\mathbb{R}^{k} \times \mathbb{R}^{N-k}$. Write $x=(y, z) \in \mathbb{R}^{k} \times \mathbb{R}^{N-k}$. We are concerned with classifying non-negative solutions of

$$
-\Delta u=\frac{u^{2^{*}(t)-1}(x)}{|y|^{t}}, \quad x \in \mathbb{R}^{N}
$$

where $0<t<\min \{2, k\}, 2^{*}(t)=\frac{2(N-t)}{N-2}$.

We will use $D^{1, p}\left(\mathbb{R}^{N}\right), 1 \leq p<N$, to denote the completion of $C_{c}^{\infty}\left(\mathbb{R}^{N}\right)$, the set of $C^{\infty}$ functions with compact support in $\mathbb{R}^{N}$, under the norm $\|u\|_{D^{1, p}\left(\mathbb{R}^{N}\right)}:=\left(\int_{\mathbb{R}^{N}}|\nabla u|^{p}\right)^{\frac{1}{p}}$. By the Gagliado-Nirenberg-Sobolev inequality,

$$
\|u\|_{L^{\frac{p N}{N-p}}\left(\mathbb{R}^{N}\right)} \leq C(N, p)\|u\|_{D^{1, p}\left(\mathbb{R}^{N}\right)} .
$$

Thus we use $D_{l o c}^{1,2}\left(\mathbb{R}^{N}\right)$ to denote those functions $u$ which satisfy, on all compact subsets $K$ of $\mathbb{R}^{N}, u \in L^{\frac{2 N}{N-2}}(K)$ and $\nabla u \in L^{2}(K)$. It is the same as $H_{l o c}^{1}\left(\mathbb{R}^{N}\right)$, another standard notation which denotes the set of functions $u$ satisfying $u, \nabla u \in L^{2}(K)$ for all compact subsets $K$ of $\mathbb{R}^{N}$.

A $D_{l o c}^{1,2}\left(\mathbb{R}^{N}\right)$ solution of (1.1) is in $L_{l o c}^{\infty}$. This can be proved by arguments similar to those used by Trudinger in [19] in proving the $L^{\infty}$ regularity of $H^{1}$ solutions to the Yamabe equation, see [8] and [16] where Hölder regularity of solutions of (1.1) were also studied. Clearly a positive solution $u$ of $(1.1)$ is $C^{\infty}$ in $\{(y, z) \mid y \neq 0\}$. See [1], [4], [5], [7], [8], [10], [11], and the references therein for related studies.

Since $u$ is superharmonic, non-negative and nonzero, it follows from the maximum principle (see, e.g. [13]) that

$$
\inf _{|x| \leq 2} u(x)>0, \quad \inf _{|x| \geq 1}\left(|x|^{N-2} u(x)\right)>0 .
$$

Our first result is

\footnotetext{
*Received July 24, 2008; accepted for publication July 31, 2008.

${ }^{\dagger}$ Institute of Applied Mathematics, AMSS, Chinese Academy of Sciences, Beijing 100080, P. R. China (dmcao@amt.ac.cn). Supported by the Fund of Distinguished Young Scholars of China and Innovative Funds of CAS in China.

${ }_{\ddagger}^{\ddagger}$ Department of Mathematics, Rutgers University, 110 Frelinghuysen Rd., Piscataway, NJ 08854, USA (yyli@math.rutgers.edu). Partially supported by NSF in USA and NSFC in China.
} 
Theorem 1.1. For $N \geq 3,2 \leq k<N$, and $t=1$, let $u \in D_{l o c}^{1,2}\left(\mathbb{R}^{N}\right) \backslash\{0\}$ be a non-negative solution of (1.1). Then

$$
u(y, z)=\mu^{\frac{N-2}{2}} u_{0}\left(\mu y, \mu z+z_{0}\right)
$$

for some $\mu>0$ and $z_{0} \in \mathbb{R}^{N-k}$, where

$$
u_{0}(y, z)=c_{N, k}\left((1+|y|)^{2}+|z|^{2}\right)^{-\frac{N-2}{2}}
$$

with $c_{N, k}=((N-2)(k-1))^{\frac{N-2}{2}}$.

Remark 1.1. If $u$ is in $D^{1,2}\left(\mathbb{R}^{N}\right)$, the result was proved by Mancini, Fabbri and Sandeep in [16]. Theorem 1.1 does not make any assumption on $u$ near infinity.

We make the following

Conjecture 1. For $N \geq 3,1 \leq k<N$ and $0<t<\min \{2, k\}$, there exists some positive $U=U_{N, k, t} \in D^{1,2}\left(\mathbb{R}^{N}\right) \cap C^{0}\left(\mathbb{R}^{N}\right)$ such that all non-negative solutions $u \in D_{\text {loc }}^{1,2}\left(\mathbb{R}^{N}\right) \backslash\{0\}$ of (1.1) must be of the form

$$
u(y, z)=\mu^{\frac{N-2}{2}} U\left(\mu y, \mu z+z_{0}\right)
$$

for some $\mu>0$ and $z_{0} \in \mathbb{R}^{N-k}$.

We present some partial results towards proving the above conjecture. In particular, we prove that $D_{\text {loc }}^{1,2}\left(\mathbb{R}^{N}\right)$ non-negative solutions of $(1.1)$ must belong to $D^{1,2}\left(\mathbb{R}^{N}\right)$. We also prove that if if we replace the exponent $2^{*}(t)-1$ by some $p<2^{*}(t)-1$, then $D_{l o c}^{1,2}\left(\mathbb{R}^{N}\right)$ non-negative solutions of the equation must be identically zero.

Theorem 1.2. For $N \geq 3,1 \leq k<N, 0<t<\min \{2, k\}$ and $p<2^{*}(t)-1$, let $u \in D_{\text {loc }}^{1,2}\left(\mathbb{R}^{N}\right)$ be a non-negative solution of

$$
-\Delta u=\frac{u^{p}(x)}{|y|^{t}}, \quad x \in \mathbb{R}^{N} .
$$

Then $u \equiv 0$.

Define for $x \in \mathbb{R}^{N}$ and $\lambda>0$ the Kelvin transform of $u$ by

$$
u_{x, \lambda}(\tilde{x})=\left(\frac{\lambda}{|\tilde{x}-x|}\right)^{N-2} u\left(x+\frac{\lambda^{2}(\tilde{x}-x)}{|\tilde{x}-x|^{2}}\right), \quad \tilde{x} \in \mathbb{R}^{N}
$$

Theorem 1.3. For $N \geq 3,1 \leq k<N$ and $0<t<\min \{2, k\}$, let $u \in$ $D_{\text {loc }}^{1,2}\left(\mathbb{R}^{N}\right) \backslash\{0\}$ be a non-negative solution of (1.1). Then

For every $x=(0, z) \in\{0\} \times \mathbb{R}^{N-k}$,

there exists $\bar{\lambda}(x) \in(0, \infty)$ such that $u_{x, \bar{\lambda}(x)} \equiv u$,

For every $\bar{x}=(\bar{y}, \bar{z}) \in\left(\mathbb{R}^{k} \backslash\{0\}\right) \times \mathbb{R}^{N-k}, 0<\lambda \leq|\bar{y}|, u_{\bar{x}, \lambda} \leq u$ in $\mathbb{R}^{N} \backslash B_{\lambda}(\bar{x})$. (1.5) Consequently,

$$
u \in D^{1,2}\left(\mathbb{R}^{N}\right),
$$


and, for some positive constant $\mu$ and some $\bar{z} \in \mathbb{R}^{N-k}$,

$$
\hat{u}(y, z):=\mu^{\frac{N-2}{2}} u(\mu y, \mu z+\bar{z})
$$

satisfies, for some constant $d>0$,

$$
\hat{u}(0, z)=\left(\frac{1}{d^{2}+|z|^{2}}\right)^{\frac{N-2}{2}}, \quad z \in \mathbb{R}^{N-k},
$$

and, with $w(s, \tau):=\hat{u}(s, 0, \cdots, 0, \tau, 0, \cdots, 0)$ where $(s, 0, \cdots, 0) \in \mathbb{R}^{k}$ and $(\tau, 0, \cdots, 0) \in \mathbb{R}^{N-k}$,

$$
\begin{gathered}
\hat{u}(y, z)=w(|y|,|z|), \quad \forall(y, z) \in \mathbb{R}^{N}, \\
\partial_{s} w(s, \tau)<0, \partial_{\tau} w(s, \tau)<0, \partial_{s}\left(s^{N-2} w(s, \tau)\right) \geq 0, \quad \forall s, \tau>0 . \\
2 s \tau \partial_{s} w(s, \tau)+\left(d^{2}+\tau^{2}-s^{2}\right) \partial_{\tau} w(s, \tau)+(N-2) \tau w(s, \tau) \equiv 0,
\end{gathered}
$$

Moreover, for $P=(-d, 0, \cdots, 0) \in \mathbb{R}^{N}$, the Kelvin transformation of $\hat{u}$,

$$
v(\tilde{x}):=\left(\frac{2 d}{|\tilde{x}-P|}\right)^{N-2} \hat{u}\left(P+\frac{4 d^{2}(\tilde{x}-P)}{|\tilde{x}-P|^{2}}\right),
$$

satisfies

$$
v\left(\tilde{y}_{1}, \tilde{y}_{2}, \cdots, \tilde{y}_{k}, \tilde{z}\right)=v\left(\sqrt{\left|\tilde{y}_{1}-d\right|^{2}+|\tilde{z}|^{2}}, \tilde{y}_{2}, \cdots, \tilde{y}_{k}, 0\right)
$$

Note that (1.8) implies

$$
w(-s, \tau) \equiv w(-s,-\tau) \equiv w(s,-\tau) \equiv w(s, t) .
$$

Note also that a standard calculation shows that the equation of $v$ is

$$
-\Delta v(\tilde{x})=\left(\frac{4 d^{2}}{|| \tilde{x}-\left.Q\right|^{2}-4 d^{2} \mid}\right)^{t} v(\tilde{x})^{2^{*}(t)-1}, \quad \text { in } \mathbb{R}^{N}
$$

where $Q=-P$.

If $k=1, v$ is a radially symmetric (with respect to $Q$ ) solution of (1.12)

The proofs of our results make use of ideas in the new proof of the Liouvilletype theorem of Caffarelli, Gidas and Spruck ([6]; see also Gidas, Ni and Nirenberg [12] for the result under some decay assumption near infinity) given in [15] and [14], which is based on the method of moving planes and full exploitation of the conformal invariance of the problem.

2. Preliminary results. In this section we present some results which will be used in the proofs of Theorem 1.1 and Theorem 1.3.

Denote $B_{r}(x)=\left\{\xi \in \mathbb{R}^{N}|| \xi-x \mid<r\right\}$ for $r>0$ and write $B_{r}(x)$ as $B_{r}$ if $x=0$.

Let $u \in D_{\text {loc }}^{1,2}\left(\mathbb{R}^{N}\right) \backslash\{0\}$ be a non-negative solution of (1.1).

Lemma 1. For any $x=(0, z) \in \mathbb{R}^{k} \times \mathbb{R}^{N-k}$, there exists $\lambda_{0}(x)>0$ such that for any $\lambda \in\left(0, \lambda_{0}(x)\right)$

$$
u_{x, \lambda} \leq u, \quad \text { in } \mathbb{R}^{N} \backslash B_{\lambda}(x)
$$


Proof. Without loss of generality, we assume $x=0$ and write $u_{0, \lambda}$ as $u_{\lambda}$. that

Step 1. We prove that there exist $0<\lambda_{1}<\lambda_{2}$, which may depend on $x$, such

$$
u_{\lambda}(\xi) \leq u(\xi), \quad \forall 0<\lambda<\lambda_{1}, \quad \lambda<|\xi|<\lambda_{2} .
$$

Indeed, for $\xi \in \partial B_{\lambda_{2}}, \frac{\lambda^{2} \xi}{|\xi|^{2}} \in B_{\lambda_{2}}$. Thus

$$
\begin{aligned}
u_{\lambda}(\xi) & =\left(\frac{\lambda}{|\xi|}\right)^{N-2} u\left(\frac{\lambda^{2} \xi}{|\xi|^{2}}\right)<\left(\frac{\lambda_{1}}{\lambda_{2}}\right)^{N-2} \sup _{\xi \in B_{\lambda_{2}}} u(\xi) \\
& \left.<\inf _{\partial B_{\lambda_{2}}} u \quad \quad \text { (by choosing } \lambda_{1}=\lambda_{1}\left(\lambda_{2}\right) \text { small }\right) \\
& \leq u(\xi) .
\end{aligned}
$$

The above inequality, together with $u_{\lambda}=u$ on $\partial B_{\lambda}$ implies that

$$
u_{\lambda} \leq u \quad \text { on } \partial\left(B_{\lambda_{2}} \backslash B_{\lambda}\right)
$$

for all $\lambda \in\left(0, \lambda_{1}\left(\lambda_{2}\right)\right)$.

We will show, for sufficiently small $\lambda_{2}$, that for $\lambda \in\left(0, \lambda_{1}\left(\lambda_{2}\right)\right)$

$$
u_{\lambda} \leq u \quad \text { in } B_{\lambda_{2}} \backslash B_{\lambda} .
$$

In the proof of (2.3) as well as in the proofs of Lemma 2 and Lemma 6, we make use of the "narrow domain technique" of Berestycki and Nirenberg [2].

For $\xi=\left(\xi_{1}, \xi_{2}\right) \in \mathbb{R}^{k} \times \mathbb{R}^{N-k}$, we have

$$
\begin{gathered}
-\Delta u(\xi)=\frac{u^{2^{*}(t)-1}(\xi)}{\left|\xi_{1}\right|^{t}}, \quad \text { in } \mathbb{R}^{N}, \\
-\Delta u_{\lambda}(\xi)=\frac{u_{\lambda}^{2^{*}(t)-1}(\xi)}{\left|\xi_{1}\right|^{t}}, \quad \text { in } \mathbb{R}^{N} \backslash\{0\},
\end{gathered}
$$

which yield

$$
\begin{aligned}
-\Delta\left(u(\xi)-u_{\lambda}(\xi)\right) & =\frac{1}{\mid \xi_{1} t^{t}}\left(u^{2^{*}(t)-1}(\xi)-u_{\lambda}^{2^{*}(t)-1}(\xi)\right) \\
& =\frac{2^{*}(t)-1}{\left|\xi_{1}\right|^{t}}\left(\theta u+(1-\theta) u_{\lambda}\right)^{2^{*}(t)-2}\left(u(\xi)-u_{\lambda}(\xi)\right) .
\end{aligned}
$$

Multiplying both sides of $(2.4)$ by $\left(u-u_{\lambda}\right)_{-}:=-\min \left\{0, u-u_{\lambda}\right\}$ we obtain

$$
\begin{aligned}
& \int_{B_{\lambda_{2}} \backslash B_{\lambda}}\left|\nabla\left(u-u_{\lambda}\right)_{-}\right|^{2} \\
= & \left(2^{*}(t)-1\right) \int_{B_{\lambda_{2} \backslash B_{\lambda}}} \frac{1}{\left|\xi_{1}\right|^{t}}\left(\theta u+(1-\theta) u_{\lambda}\right)^{2^{*}(t)-2}\left|\left(u(\xi)-u_{\lambda}(\xi)\right)_{-}\right|^{2} \\
\leq & \left(2^{*}(t)-1\right)\left(\int_{B_{\lambda_{2}} \backslash B_{\lambda}}\left(\theta u+(1-\theta) u_{\lambda}\right)^{\frac{2 N}{N-2}}\right)^{\frac{2-t}{N}}\left(\int_{B_{\lambda_{2}} \backslash B_{\lambda}} \frac{\left|\left(u-u_{\lambda}\right)_{-}\right|^{\frac{2 N}{N+t-2}}}{\left|\xi_{1}\right|^{\frac{t N}{N+t-2}}}\right)^{\frac{N+t-2}{N}} \\
\leq & C\left(\int_{B_{\lambda_{2}} \backslash B_{\lambda}}\left(|u|+\left|u_{\lambda}\right|\right)^{\frac{2 N}{N-2}}\right)^{\frac{2-t}{N}} \int_{B_{\lambda_{2}} \backslash B_{\lambda}}\left|\nabla\left(u-u_{\lambda}\right)_{-}\right|^{2} .
\end{aligned}
$$


Suppose that $\int_{B_{\lambda_{2}} \backslash B_{\lambda}}\left|\nabla\left(u-u_{\lambda}\right)_{-}\right|^{2} \neq 0$ then we have, for some constant $C$ independent of $\lambda_{2}$,

$$
1 \leq C\left(\int_{B_{\lambda_{2} \backslash B_{\lambda}}}\left(|u|+\left|u_{\lambda}\right|\right)^{\frac{2 N}{N-2}}\right)^{\frac{2-t}{N}} \leq C\left(\int_{B_{\lambda_{2}}}|u|^{\frac{2 N}{N-2}}\right)^{\frac{2-t}{N}},
$$

which is a contradiction if $\lambda_{2}$ is chosen to be small enough.

Thus, for small $\lambda_{2}, \int_{B_{\lambda_{2}} \backslash B_{\lambda}}\left|\nabla\left(u-u_{\lambda}\right)_{-}\right|^{2}=0$. So $\left(u-u_{\lambda}\right)_{-} \equiv 0$ on $B_{\lambda_{2}} \backslash B_{\lambda}$ and (2.3) is proved.

The values of $\lambda_{1}$ and $\lambda_{2}$ are fixed by now.

Step 2. We show that for some small $\lambda_{0}(x) \in\left(0, \lambda_{1}\right)$,

$$
u_{\lambda}(\xi) \leq u(\xi), \quad \forall 0<\lambda<\lambda_{0}(x), \quad|\xi| \geq \lambda_{2} .
$$

Let $\varphi(\xi)=\left(\frac{\lambda_{2}}{|\xi|}\right)^{N-2} \inf _{\partial B_{\lambda_{2}}} u$. Since $\varphi$ is harmonic on $\mathbb{R}^{N} \backslash\{0\}$ and $\varphi \leq u$ when $|\xi|=\lambda_{2}$ we have

$$
u(\xi) \geq\left(\frac{\lambda_{2}}{|\xi|}\right)^{N-2} \inf _{\partial B_{\lambda_{2}}} u, \quad|\xi| \geq \lambda_{2} .
$$

Denote $\lambda_{0}=\inf \left\{\lambda_{1}, \lambda_{2}\left(\frac{\inf _{\partial B_{\lambda_{2}}} u}{\sup _{B_{\lambda_{2}}} u}\right)^{\frac{1}{N-2}}\right\}$. For any $0<\lambda<\lambda_{0},|\xi| \geq \lambda_{2}$ we have

$$
u_{\lambda}(\xi)=\left(\frac{\lambda}{|\xi|}\right)^{N-2} u\left(\frac{\lambda^{2} \xi}{|\xi|^{2}}\right) \leq\left(\frac{\lambda_{0}}{|\xi|}\right)^{N-2} \sup _{B_{\lambda_{2}}} u \leq\left(\frac{\lambda_{2}}{|\xi|}\right)^{N-2} \inf _{\partial B_{\lambda_{2}}} u \leq u(\xi),
$$

where in the last inequality (2.6) has been used. Lemma 1 is thus proved.

With Lemma 1, we can define, for $x=(0, z) \in \mathbb{R}^{k} \times \mathbb{R}^{N-k}$,

$$
\bar{\lambda}(x)=\sup \left\{\mu>0 \mid u_{x, \lambda} \leq u \text { in } \mathbb{R}^{N} \backslash B_{\lambda}(x), \forall 0<\lambda \leq \mu\right\} .
$$

Lemma 2. If $\bar{\lambda}(x)<\infty$ for some $x=(0, z) \in \mathbb{R}^{k} \times \mathbb{R}^{N-k}$, then

$$
u \equiv u_{x, \bar{\lambda}(x)} .
$$

Proof. Without loss of generality we assume $x=0$ and denote $u_{0, \lambda}$ by $u_{\lambda}$ and $\bar{\lambda}(0)$ by $\bar{\lambda}$. By the definition of $\bar{\lambda}(0)$ we know

$$
u_{\bar{\lambda}} \leq u \quad \text { in } \mathbb{R}^{N} \backslash B_{\bar{\lambda}} .
$$

This is equivalent to

$$
u_{\bar{\lambda}} \geq u \quad \text { in } B_{\bar{\lambda}} .
$$

If (2.7) does not hold, then by the strong maximum principle

$$
\inf _{B_{\bar{\lambda}-\epsilon^{\prime}} \backslash\left(\{0\} \times \mathbb{R}^{N-k}\right)}\left(u_{\bar{\lambda}}-u\right)>0, \quad \forall 0<\epsilon^{\prime}<\bar{\lambda} .
$$


To see (2.8) we make use of

$$
\begin{gathered}
-\Delta u(\xi)=\frac{1}{\left|\xi_{1}\right| t} u^{2^{*}(t)-1}(\xi), \quad \xi=\left(\xi_{1}, \xi_{2}\right) \in B_{\bar{\lambda}}, \\
-\Delta u_{\bar{\lambda}}(\xi)=\frac{1}{\left|\xi_{1}\right|^{t}} u_{\bar{\lambda}}^{2^{*}(t)-1}(\xi), \quad \xi=\left(\xi_{1}, \xi_{2}\right) \in B_{\bar{\lambda}} \backslash\{0\} .
\end{gathered}
$$

Using $u_{\bar{\lambda}} \geq u$, and using the fact that $\{0\}$ has zero (Newtonian) capacity, we obtain in the sense of distribution that

$$
\Delta\left(u-u_{\bar{\lambda}}\right) \geq 0, \quad \text { in } B_{\bar{\lambda}} .
$$

So if $u-u_{\bar{\lambda}} \equiv 0$ does not hold, we must have (2.8). So, for $0<\delta<\bar{\lambda}$, there exists some $c(\delta)>0$ such that

$$
\inf _{K_{\delta}}\left(u_{\bar{\lambda}}-u\right) \geq c(\delta)
$$

where

$$
K_{\delta}=\left\{x \in B_{\bar{\lambda}} \mid \operatorname{dist}\left(x, \partial\left(B_{\bar{\lambda}} \backslash\left(\{0\} \times \mathbb{R}^{N-k}\right)\right)\right) \geq \delta\right\} .
$$

By the uniform continuity of $u$ on the compact set $K_{\delta}$, there exists small $\epsilon_{1}=\epsilon(\delta) \epsilon$ $(0, \delta)$ such that

$$
u_{\lambda}-u \geq c(\delta) / 2, \quad \text { on } K_{2 \delta}, \quad \forall \bar{\lambda} \leq \lambda \leq \bar{\lambda}+\epsilon_{1} .
$$

Using the "narrow domain technique" as in the proof of Lemma 1, we can show that for some small $\delta>0$,

$$
u_{\lambda}-u \geq 0, \quad \text { on } B_{\lambda} \backslash K_{2 \delta}, \quad \forall \bar{\lambda} \leq \lambda \leq \bar{\lambda}+\epsilon_{1} .
$$

This and (2.11) lead to a contradiction to the definition of $\bar{\lambda}$. Lemma 2 is established. $\square$

Lemma 3. Either $\bar{\lambda}(x)=\infty$ for all $x=(0, z)$ in $\{0\} \times \mathbb{R}^{N-k}$ or $\bar{\lambda}(x)<\infty$ for all $x=(0, z)$ in $\{0\} \times \mathbb{R}^{N-k}$.

Proof. Suppose that there is a point $\bar{x}=(0, \bar{z}) \in \mathbb{R}^{k} \times \mathbb{R}^{N-k}$, such that $0<$ $\bar{\lambda}(\bar{x})<\infty$, then by Lemma 2

$$
u(\xi) \equiv\left(\frac{\bar{\lambda}(\bar{x})}{|\xi-\bar{x}|}\right)^{N-2} u\left(\bar{x}+\frac{\bar{\lambda}^{2}(\bar{x})(\xi-\bar{x})}{|\xi-\bar{x}|^{2}}\right) .
$$

Multiplying (2.12) by $|\xi|^{N-2}$ and sending $|\xi|$ to $\infty$, we obtain

$$
\lim _{|\xi| \rightarrow \infty}|\xi|^{N-2} u(\xi)=\bar{\lambda}^{N-2}(\bar{x}) u(\bar{x})<\infty .
$$

On the other hand, for all $x=(0, z)$,

$$
u_{x, \lambda}(\xi) \leq u(\xi), \quad \forall|\xi-x|>\lambda, 0<\lambda<\bar{\lambda}(x),
$$


leading to

$$
\lim \inf _{|\xi| \rightarrow \infty}|\xi|^{N-2} u(\xi) \geq \lambda^{N-2} u(x), \quad \forall 0<\lambda<\bar{\lambda}(x) .
$$

Letting $\lambda \rightarrow \bar{\lambda}(x)$ in (2.14), we have

$$
\lim \inf _{|\xi| \rightarrow \infty}|\xi|^{N-2} u(\xi) \geq \bar{\lambda}^{N-2}(x) u(x),
$$

which implies, in view of $(2.13)$, that $\bar{\lambda}(x)<\infty$ for all $x=(0, z)$. Lemma 3 is established. $\square$

Lemma 4. For $1 \leq k<N, \bar{y} \neq 0, \bar{x}=(\bar{y}, \bar{z}) \in \mathbb{R}^{k} \times \mathbb{R}^{N-k}$, and $\lambda \in(0,|\bar{y}|)$, we have the following inequality

$$
\left(\frac{\lambda}{|x-\bar{x}|}\right)^{2} \frac{|y|}{\left|\bar{y}+\frac{\lambda^{2}(y-\bar{y})}{|x-\bar{x}|^{2}}\right|} \geq 1, \quad \forall 0<|x-\bar{x}| \leq \lambda, x=(y, z) \in \mathbb{R}^{k} \times \mathbb{R}^{N-k}
$$

Proof. It is easy to see that (2.15) is equivalent to

$$
\lambda^{2}|y| \geq\left|\lambda^{2} y+\left(|x-\bar{x}|^{2}-\lambda^{2}\right) \bar{y}\right|
$$

which is equivalent to

$$
-2 \lambda^{2}\langle y, \bar{y}\rangle \leq\left(\lambda^{2}-|x-\bar{x}|^{2}\right)|\bar{y}|^{2}
$$

which is equivalent to

$$
-2 \lambda^{2}\langle y-\bar{y}, \bar{y}\rangle \leq\left(3 \lambda^{2}-|x-\bar{x}|^{2}\right)|\bar{y}|^{2} .
$$

Inequality (2.16) follows from the following simple calculation:

$$
-2 \lambda^{2}\langle y-\bar{y}, \bar{y}\rangle \leq 2 \lambda^{2}|y-\bar{y}||\bar{y}| \leq 2 \lambda^{2}(\lambda|\bar{y}|) \leq 2 \lambda^{2}|\bar{y}|^{2} \leq\left(3 \lambda^{2}-|x-\bar{x}|^{2}\right)|\bar{y}|^{2} .
$$

Lemma 4 is established.

Lemma 5. Let $\bar{x}$ be as in Lemma 4. Then there exists $\lambda_{0}(\bar{x}) \in(0,|\bar{y}|)$ such that for any $\lambda \in\left(0, \lambda_{0}(\bar{x})\right)$

$$
u_{\bar{x}, \lambda}(x) \leq u(x), \quad \forall \xi \in \mathbb{R}^{N} \backslash B_{\lambda}(\bar{x}) .
$$

Proof. Given (1.2), and given the smoothness of $u$ near $\bar{x}$, the proof is the same as that of lemma 2.1 in [14].

Let

$$
\bar{\lambda}(\bar{x}):=\sup \left\{\mu|0<\mu<| \bar{y} \mid, u_{\bar{x}, \lambda} \leq u \text { in } \mathbb{R}^{N} \backslash B_{\lambda}(\bar{x}),, \forall 0<\lambda \leq \mu\right\} .
$$

Lemma 6. Let $\bar{x}=(\bar{y}, \bar{z})$ be as in Lemma 4. Then $\bar{\lambda}(\bar{x})=|\bar{y}|$. Namely

$$
u_{\bar{x}, \lambda}(x) \leq u(x), \quad \forall x=(y, z) \in \mathbb{R}^{N} \backslash B_{\lambda}(\bar{x}), \quad \forall 0<\lambda \leq|\bar{y}| .
$$


Consequently, with $\tilde{w}(s, z):=u(s, 0, \cdots, 0, z)$,

$$
\begin{gathered}
u(y, z)=\tilde{w}(|y|, z), \quad \forall(y, z) \in \mathbb{R}^{N}, \\
\partial_{s} \tilde{w}(s, z)<0, \quad \partial_{s}\left(s^{N-2} \tilde{w}(s, z)\right) \geq 0, \quad \forall s>0, z \in \mathbb{R}^{N-k} .
\end{gathered}
$$

Proof. Without loss of generality we assume that $\bar{z}=0$. We prove it by contradiction argument. Suppose the contrary, then $0<\bar{\lambda}(\bar{x})<|\bar{y}|$. Using Lemma 4 we have, for any $\lambda \in(0,|\bar{y}|)$,

$$
-\Delta u_{\bar{x}, \lambda}(x) \geq \frac{1}{|y|^{t}} u_{\bar{x}, \lambda}(x)^{2^{*}(t)-1}, \quad \forall 0<|x-\bar{x}|<\lambda .
$$

Indeed, for $|x-\bar{x}|<\lambda$,

$$
\begin{aligned}
& -\Delta u_{\bar{x}, \lambda}(x)=\left(\frac{\lambda}{|x-\bar{x}|}\right)^{N+2}(-\Delta u)\left(\bar{x}+\frac{\lambda^{2}(x-\bar{x})}{|x-\bar{x}|^{2}}\right) \\
& =\left(\frac{\lambda}{|x-\bar{x}|}\right)^{N+2} \frac{1}{\left|\bar{y}+\frac{\lambda^{2}(y-\bar{y})}{|x-\bar{x}|^{2}}\right|^{t}} u^{2^{*}(t)-1}\left(\bar{x}+\frac{\lambda^{2}(x-\bar{x})}{|x-\bar{x}|^{2}}\right)
\end{aligned}
$$

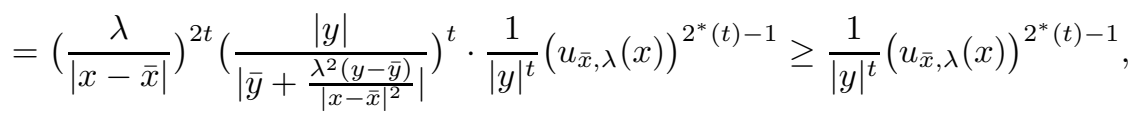

where in the last inequality Lemma 4 has been used.

By the definition of $\bar{\lambda}(\bar{x})$,

$$
u-u_{\bar{x}, \bar{\lambda}(\bar{x})} \geq 0, \quad \text { in } \mathbb{R}^{N} \backslash B_{\bar{\lambda}(\bar{x})}(\bar{x}) .
$$

This is equivalent to

$$
u_{\bar{x}, \bar{\lambda}(\bar{x})}-u \geq 0, \quad \text { in } B_{\bar{\lambda}(\bar{x})}(\bar{x}) \backslash\{\bar{x}\} .
$$

With this, we derive from (2.20) and the equation of $u$ that

$$
\Delta\left(u_{\bar{x}, \bar{\lambda}(\bar{x})}-u\right) \leq 0, \quad \text { in } B_{\bar{\lambda}(\bar{x})}(\bar{x}) \backslash\{\bar{x}\} .
$$

Since $\{\bar{x}\}$ has zero (Newtonian) capacity, we deduce from this and (2.21) that

$$
\Delta\left(u_{\bar{x}, \bar{\lambda}(\bar{x})}-u\right) \leq 0, \quad \text { in } B_{\bar{\lambda}(\bar{x})}(\bar{x}), \quad \text { in the distribution sense. }
$$

Since $\bar{\lambda}(\bar{x})<|\bar{y}|$, both $u$ and $u_{\bar{x}, \bar{\lambda}(\bar{x})}$ are smooth near $\partial B_{\bar{\lambda}(\bar{x})}(\bar{x})$. Also, $u_{\bar{x}, \bar{\lambda}(\bar{x})}$ is smooth near $\left\{(0, z) \mid z \in \mathbb{R}^{N-k}\right\}$, and therefore, in view of the equation of $u, u_{\bar{x}, \bar{\lambda}(\bar{x})}$ can not be equal to $u$. Thus, by the maximum principle,

$$
\inf _{B_{\bar{\lambda}(\bar{x})-\epsilon^{\prime}}(\bar{x})}\left(u_{\bar{x}, \bar{\lambda}(\bar{x})}-u\right)>0, \quad \forall 0<\epsilon^{\prime}<\bar{\lambda}(\bar{x}) .
$$

With the above information, essentially the same arguments as in the proof of Lemma 1 and Lemma 2 yields, for some $\epsilon>0, u_{\bar{x}, \lambda} \geq u$ in $B_{\lambda}(\bar{x}) \backslash\{\bar{x}\}$ for all $\bar{\lambda} \leq \lambda \leq \bar{\lambda}+\epsilon$. 
This is equivalent to $u \geq u_{\bar{x}, \lambda}$ in $\mathbb{R}^{N} \backslash B_{\lambda}(\bar{x})$ for all $\bar{\lambda} \leq \lambda \leq \bar{\lambda}+\epsilon$, which violates the definition of $\bar{\lambda}$. (2.17) is proved.

For $y_{1}<0<\bar{y}_{1}, \bar{y}=\left(\bar{y}_{1}, 0, \cdots, 0\right), \lambda=|\bar{y}|=\bar{y}_{1},(2.17)$ with $y=\left(y_{1}, 0, \cdots, 0\right)$, $z \in \mathbb{R}^{N-k}$ and $x=(y, z)$ leads to, after sending $\bar{y}_{1} \rightarrow \infty$,

$$
u\left(-y_{1}, 0, \cdots, 0, z\right) \leq u\left(y_{1}, 0, \cdots, 0, z\right) .
$$

By the symmetry of the problem, $u_{O}(y, z):=u(O y, z)$ satisfies the same equation for all orthogonal matrix $O \in O(k)$, so we also have

$$
u_{O}\left(-y_{1}, 0, \cdots, 0, z\right) \leq u_{O}\left(y_{1}, 0, \cdots, 0, z\right),
$$

which implies that $u$ is radially symmetric about the origin in the $y$-variables. Namely (2.18) holds.

For $0<y_{1}<a<\bar{y}_{1}, \bar{y}=\left(\bar{y}_{1}, 0, \cdots, 0\right), \bar{x}=(\bar{y}, 0), \hat{y}=(a, 0, \cdots, 0), \lambda=|\bar{y}-\hat{y}|=$ $\bar{y}_{1}-a,(2.17)$ with $y=\left(y_{1}, 0, \cdots, 0\right), z \in \mathbb{R}^{N-k}$ and $x=(y, z)$ leads to, after sending $\bar{y}_{1} \rightarrow \infty$,

$$
u\left(2 a-y_{1}, 0, \cdots, 0, z\right) \leq u\left(y_{1}, 0, \cdots, 0, z\right), \quad \forall z \in \mathbb{R}^{N-1} .
$$

which implies $\tilde{w}(s, z) \leq \tilde{w}(\tau, z)$ for $s \geq \tau>0, z \in \mathbb{R}^{N-k}$. Thus $\partial_{s} \tilde{w}(s, z) \leq 0$. Since $\partial_{s} \tilde{w}$ satisfies a linear second order elliptic partial differential equation, by applying $\partial_{s}$ to the equation satisfied by $\tilde{w}$, and since $\tilde{w}$ must depend on $s$, a fact easily seen from the equation of $u$, we obtain the first inequality in (2.19) by using the strong maximum principle.

For $\frac{s}{2}<y_{1}<s<\hat{y}_{1}$, let $\lambda=s-y_{1} \in\left(0, y_{1}\right), y=\left(y_{1}, 0, \cdots, 0\right), x=(y, 0), \hat{y}=$ $\left(\hat{y}_{1}, 0, \cdots, 0\right), \hat{x}=(\hat{y}, 0)$. Clearly $|\hat{x}-x|=\hat{y}_{1}-y_{1}>\lambda$. By Lemma $6, u_{x, \lambda}(\hat{x}) \leq u(\hat{x})$, i.e.

$$
\left(\frac{\hat{y}_{1}-y_{1}}{\lambda}\right)^{N-2} \tilde{w}\left(\hat{y}_{1}, 0\right)-\tilde{w}\left(y_{1}+\frac{\lambda^{2}}{\hat{y}_{1}-y_{1}}, 0\right) \geq 0 .
$$

Since the left hand side is equal to 0 for $\hat{y}_{1}=s=y_{1}+\lambda$, so the derivative of the left hand side in $\hat{y}_{1}$ at $s$ is $\geq 0$. Namely

$$
2 \partial_{s} \tilde{w}(s, 0)+\frac{N-2}{s-y_{1}} \tilde{w}(s, 0) \geq 0 .
$$

Sending $y_{1}$ to $\frac{s}{2}$ in the above leads to

$$
\frac{\partial}{\partial s}\left(s^{N-2} \tilde{w}(s, 0)\right)=s^{N-2}\left(\partial_{s} \tilde{w}(s, 0)+\frac{N-2}{s} s \tilde{w}(s, 0)\right) \geq 0, \quad \forall s>0 .
$$

Since the problem is invariant under translation in $z$-variables, we obtain the second inequality in (2.19). Lemma 6 is established.

Lemma 7. For $\alpha>0, \theta \in(0,1]$,

$$
-f^{\prime \prime}(y)=\frac{1}{y^{\theta}} f(y)^{\alpha}, \quad f(y)>0, \quad y>0
$$

has no solution. 
Proof of Lemma \%. We write $h(f)=f^{\alpha}$. First we claim that

$$
f^{\prime}(y)>0, \quad \text { for any } y>0 .
$$

Indeed, if for some $y_{0}>0$ we have $f^{\prime}\left(y_{0}\right) \leq 0$, then by the mean value theorem

$$
f^{\prime}(y)=f^{\prime}\left(y_{0}\right)+f^{\prime \prime}(\hat{y})\left(y-y_{0}\right)<0, \quad \forall y>y_{0} .
$$

So for all $y>y_{1}>y_{0}$,

$$
f^{\prime}(y)<f^{\prime}\left(y_{1}\right)<0
$$

and therefore

$$
f(y)=f\left(y_{1}\right)+\int_{y_{1}}^{y} f^{\prime}(s) d s \leq f\left(y_{1}\right)+f^{\prime}\left(y_{1}\right)\left(y-y_{1}\right) .
$$

Sending $y$ to $\infty$ and using $f^{\prime}\left(y_{1}\right)<0$ we have

$$
f(y) \rightarrow-\infty \quad \text { as } y \rightarrow \infty,
$$

contradicting the positivity of $f$.

Now since $f$ is increasing and $h$ is non-decreasing

$$
h(f(t)) \geq h(f(s)), \forall t>s>0 .
$$

Thus

$$
-f^{\prime \prime}(t)=\frac{1}{t^{\theta}} f^{\alpha}(t) \geq \frac{1}{t^{\theta}} f^{\alpha}(s), \forall t>s>0 .
$$

It follows by integrating over $(s, t)$ and using $f^{\prime}>0$, we have

$$
f^{\prime}(s) \geq f^{\prime}(s)-f^{\prime}(t) \geq h(f(s)) \int_{s}^{t} y^{-\theta} d y
$$

Sending $t$ to $\infty$, we get $f^{\prime}(s) \geq \infty$. A contradiction.

Lemma 8. For $\alpha>0, t<2$,

$$
\left\{\begin{array}{l}
-\frac{1}{r}\left(r f^{\prime}\right)^{\prime}=\frac{1}{r^{t}} f^{\alpha}, \quad f(r)>0, \quad r>0 \\
\int_{0}^{1} r\left(f^{\prime}(r)\right)^{2}<\infty
\end{array}\right.
$$

has no solution.

Proof. We first show that

$$
\lim _{r \rightarrow 0^{+}}\left(r f^{\prime}(r)\right)=0 .
$$

Since $\left(r f^{\prime}\right)^{\prime}<0, \lim _{r \rightarrow 0^{+}}\left(r f^{\prime}(r)\right)$ exists. If the limit is not 0 , then there exists some constant $b>0$ such that $r\left|f^{\prime}(r)\right|>b$ for small $r>0$. It follows that $r\left|f^{\prime}(r)\right|^{2} \geq$ $b^{2} r^{-1}$ for small $r$, violating $\int_{0}^{1} r\left(f^{\prime}(r)\right)^{2}<\infty$. (2.26) is proved. 
Since $\left(r f^{\prime}\right)^{\prime}<0$, we know from $(2.26)$ that

$$
f^{\prime}(r)<0 \quad \forall 0<r<\infty \text {. }
$$

Integrating the equation of $f$, we have, in view of (2.26),

$$
-r f^{\prime}(r)=\int_{0}^{r} s^{1-t} f(s)^{\alpha} d s
$$

Another integration gives

$$
f(1) \geq f(1)-f(R)=\int_{1}^{R} \int_{0}^{r} r^{-1} s^{1-t} f(s)^{\alpha} d s d r, \quad \forall 1<R<\infty .
$$

Sending $R$ to $\infty$ in the above gives

$$
\int_{1}^{\infty} \int_{0}^{r} r^{-1} s^{1-t} f(s)^{\alpha} d s d r<\infty
$$

Interchanging the order of integration (Fubini theorem), we obtain

$$
\int_{0}^{1} \int_{1}^{\infty} r^{-1} s^{1-t} f(s)^{\alpha} d r d s<\infty
$$

A contradiction. Lemma 8 is established.

LEMMA 9. (1.4) holds.

Proof. By Lemma 3, we only need to rule out the possibility that $\bar{\lambda}(x)=\infty$ for all $x=(0, z) \in\{0\} \times \mathbb{R}^{N-k}$. Indeed if this occurs, then, by lemma 11.3 of [14],

$$
u(y, z)=u(y, 0), \quad \forall y \in \mathbb{R}^{k} .
$$

By Lemma $6, u(y, z)$ is radially symmetric in the $y$-variables, so $v(y):=u(y, 0)$ is a radially symmetric positive solution of

$$
-\Delta v(y)=\frac{v^{2^{*}(t)-1}}{|y|^{t}}, \quad y \in \mathbb{R}^{k}
$$

We also know that

$$
\int_{|y| \leq 1}\left(|\nabla v|^{2}+v^{\frac{2 N}{N-2}}\right)<\infty
$$

For $k=1$, we know from Lemma 7 that (2.28) has no positive radially symmetric solution.

For $k=2$, we know from Lemma 8 that (2.28) has no positive radially symmetric solution satisfying $(2.29)$.

For $3 \leq k<N$, (2.28) has no positive radially symmetric solution satisfying (2.29). Indeed, let

$$
\ell=\frac{k+2-2 t}{k-2}, p=2^{*}(t)-1, \alpha=\frac{2-t}{p-1}
$$


It is easy to check that $p<\ell$. Thus, according to proposition 5.2 in [18] by Serrin and Zou, radially symmetric positive solutions of (2.28), if any, satisfy $\lim _{y \rightarrow 0}|y|^{\alpha} u(y)=$ $\lambda$, for some positive constant $\lambda$. But $\alpha\left(\frac{2 N}{N-2}\right)=N$, and therefore $u$ does not satisfy (2.29). This leads to a contradiction. Lemma 9 is established.

Lemma 10. There exist some $\mu>0$ and $\bar{z} \in \mathbb{R}^{N-k}$ such that $\hat{u}$, defined by (1.6), is radially symmetric in the $z$-variables. Moreover, for $w$ defined in Theorem 1.3, $\partial_{\tau} w(s, \tau)<0, \forall s, \tau>0$.

Proof. Applying lemma 11.1 in [14] to $u(0, z)$ we know that there exist $a \geq 0, \tilde{d}>0$ and $\bar{z} \in \mathbb{R}^{N-k}$ such that

$$
u(0, z)=\left(\frac{a}{\tilde{d}^{2}+|z-\bar{z}|^{2}}\right)^{\frac{N-2}{2}} .
$$

For $\mu=a$,

$$
\hat{u}(y, z):=\mu^{\frac{N-2}{2}} u(\mu y, \mu z+\bar{z})
$$

satisfies the same equation as $u$, and

$$
\hat{u}(0, z)=\left(\frac{1}{d^{2}+|z|^{2}}\right)^{\frac{N-2}{2}},
$$

where $d=a^{-1} \tilde{d}$.

By Lemma 9, applied to $\hat{u}$, (1.4) holds for $\hat{u}$. For any $x=(0, z)$, multiplying $\hat{u}_{x, \bar{\lambda}(x)}(\tilde{x}) \equiv \hat{u}(\tilde{x})$ by $|\tilde{x}|^{N-2}$ and sending $|\tilde{x}|$ to infinity lead to, in view of $(2.31)$

$$
\bar{\lambda}(x)=\hat{u}(x)^{-\frac{1}{N-2}}=\sqrt{d^{2}+|z|^{2}} .
$$

For $\tilde{z}_{1}<0<z_{1}, \tilde{z}=\left(\tilde{z}_{1}, 0, \cdots, 0\right), \tilde{y} \in \mathbb{R}^{k}, \tilde{x}=(\tilde{y}, \tilde{z}), z=\left(z_{1}, 0, \cdots, 0\right), x=(0, z) \in$ $\mathbb{R}^{N}$, we have, in view of Lemma 9 and (2.32),

$$
\hat{u}_{x, \bar{\lambda}(x)}(\tilde{x})=\hat{u}(\tilde{x}),
$$

where $\bar{\lambda}(x)=\sqrt{d^{2}+\left|z_{1}\right|^{2}}$.

Sending $z_{1}$ to $\infty$ in the above leads to

$$
\hat{u}\left(\tilde{y},-\tilde{z}_{1}, 0, \cdots, 0\right)=\hat{u}\left(\tilde{y}, \tilde{z}_{1}, 0, \cdots, 0\right) .
$$

Namely $\hat{u}(y, z)$ is symmetric with respect to the hyperplane plane $\left\{z_{1}=0\right\}$. Since the problem is rotationally invariant with respect to the $z$-variables, we obtain the radial symmetry of $\hat{u}$ in the $z$-variables.

For $a>0,0<\tilde{z}_{1}<a<z_{1}, \tilde{y} \in \mathbb{R}^{k}, \tilde{x}=(\tilde{y}, \tilde{z}), z=\left(z_{1}, 0, \cdots, 0\right), x=(0, z) \in \mathbb{R}^{N}$, we have

$$
\hat{u}_{x, \lambda}(\tilde{x}) \leq \hat{u}(\tilde{x})
$$

where $\lambda=\left|z_{1}-a\right|<\bar{\lambda}(x)=\sqrt{d^{2}+\left|z_{1}\right|^{2}}$. Sending $z_{1}$ to $\infty$, we have

$$
\hat{u}\left(\tilde{y}, 2 a-\tilde{z}_{1}, 0, \cdots, 0\right) \leq \hat{u}\left(\tilde{y}, \tilde{z}_{1}, 0, \cdots, 0\right) .
$$

It follows that $\partial_{\tau} w(s, \tau) \leq 0$ for $s, \tau>0$. Since $\partial_{\tau} w(s, \tau)$ satisfies a linear second order elliptic partial differential equation, we deduce by using the strong maximum principle that $\partial_{\tau} w(s, \tau)>0$ for $s, \tau>0$. Lemma 10 is established. 
3. Proofs of Theorem 1.1 and Theorem 1.3. First we give

Proof of Theorem 1.1. By Lemma 9, (1.4) holds. Thus $u \in D^{1,2}\left(\mathbb{R}^{N}\right)$, and the result follows from [16]. Theorem 1.1 is established. $\square$

Now we give the

Proof of Theorem 1.3. We have proved in the last section that (1.4), (1.5), (1.2), (1.7), (1.8), (1.9), and $u \in D^{1,2}\left(\mathbb{R}^{N}\right)$. As shown in the proof of Lemma 10 , for some $\mu>0$ and $\bar{z} \in \mathbb{R}^{N-k}, \hat{u}$ defined in (2.30) satisfies (2.31). Furthermore, for any $x=(0, z)$,

$$
\hat{u}_{x, \bar{\lambda}(x)} \equiv \hat{u},
$$

where $\bar{\lambda}(x)$ is given in $(2.32)$.

Let $P_{1}=(-d, 0, \cdots, 0) \in \mathbb{R}^{k}, P=\left(P_{1}, 0\right) \in \mathbb{R}^{N}$, and $Q=\left(Q_{1}, 0\right)=-P$.

By $(2.32)$, the spheres $\left\{\partial B_{\bar{\lambda}(x)}(x)\right\}_{x=(0, z) \in\{0\} \times \mathbb{R}^{N-k}}$ are exactly the set of spheres going through the $(k-1)$-dimensional sphere $\left\{(y, 0) \in \mathbb{R}^{k} \times \mathbb{R}^{N-k}|| y \mid=d\right\} \in$ $\mathbb{R}^{k} \times\{0\}$.

Consider the Kelvin transformation of $\hat{u}$ with respect to the Möbius transformation $x \rightarrow P+\frac{4 d^{2}(x-P)}{|x-P|^{2}}$ which maps $B_{2 d}(P)$ to $\left(\mathbb{R}^{N} \backslash \overline{B_{2 d}(P)}\right) \cup\{\infty\}$ :

$$
v(\tilde{x}):=\left(\frac{2 d}{|\tilde{x}-P|}\right)^{N-2} \hat{u}\left(P+\frac{4 d^{2}(\tilde{x}-P)}{|\tilde{x}-P|^{2}}\right) .
$$

We know from (3.33) that $\hat{u}$ is symmetric with respect to $\left\{\partial B_{\bar{\lambda}(x)}(x)\right\}_{x=(0, z) \in\{0\} \times \mathbb{R}^{N-k}}$, and the Möbius transformation maps the $(k-1)$-dimensional sphere $\left\{(y, 0) \in \mathbb{R}^{k} \times \mathbb{R}^{N-k}|| y \mid=d\right\} \in \mathbb{R}^{k} \times\{0\}$ to the $(k-1)$-dimensional plane

$$
M^{k-1}=\left\{(\tilde{y}, 0) \in \mathbb{R}^{k} \times \mathbb{R}^{N-k} \mid\left(\tilde{y}-Q_{1}\right) \cdot Q_{1}=0\right\} .
$$

For $k=1, M^{0}=\{Q\}$; while for $2 \leq k<N, M^{k-1}=\left\{\left(0, y_{2}, \cdots, y_{k}, 0\right)\right\}$.

If $k=1$, then $v$ is, see e.g. [3], symmetric with respect to all hyperplanes in $\mathbb{R}^{N}$ which go through $Q$. Thus $v$ is radially symmetric with respect to $Q$, i.e. $v(\tilde{x})=v(\hat{x})$ if $|\tilde{x}-Q|=|\hat{x}-Q|$.

If $k \geq 2$, then $v$ is symmetric with respect to all hyperplanes in $\mathbb{R}^{N}$ which go through $M^{k-1}$. Thus $v$ satisfies (1.11).

Now we prove (1.10). Write $\hat{w}=w^{-2 /(N-2)}$. For $s, \tau, \tilde{s}, \tilde{\tau} \in \mathbb{R}$, let $z=$ $(\tau, 0, \cdots, 0), x=(0, z), \tilde{y}=(\tilde{s}, 0, \cdots, 0), \tilde{z}=(\tilde{\tau}, 0, \cdots, 0), \tilde{x}=(\tilde{y}, \tilde{z})$. By $(3.33)$, evaluated at $\tilde{x}$, i.e.

$$
\left(\frac{|\tilde{s}|^{2}+|\tilde{\tau}-\tau|^{2}}{d^{2}+|\tau|^{2}}\right) \hat{w}\left(\frac{\left(d^{2}+|\tau|^{2}\right) \tilde{s}}{|\tilde{s}|^{2}+|\tilde{\tau}-\tau|^{2}}, \tau+\frac{\left(d^{2}+|\tau|^{2}\right)(\tilde{\tau}-\tau)}{|\tilde{s}|^{2}+|\tilde{\tau}-\tau|^{2}}\right) \equiv \hat{w}(\tilde{s}, \tilde{\tau}) .
$$

For $(\tilde{s}, \tilde{\tau}), \tilde{\tau} \neq 0$, let

$$
\tau^{*}=\tau^{*}(\tilde{s}, \tilde{\tau})=\frac{\tilde{s}^{2}+\tilde{\tau}^{2}-d^{2}}{2 \tilde{\tau}}
$$

It is easy to see that

$$
|\tilde{s}|^{2}+\left|\tilde{\tau}-\tau^{*}(\tilde{s}, \tilde{\tau})\right|^{2}=d^{2}+|\tau|^{2},
$$




$$
\tilde{\tau}-\tau=\frac{d^{2}+\tilde{\tau}^{2}-\tilde{s}^{2}}{2 \tilde{\tau}} .
$$

Applying $\frac{\partial}{\partial \tau}$ to $(3.34)$ and evaluating it at $\tau=\tau^{*}(\tilde{s}, \tilde{\tau})$ lead to

$$
-\hat{w}(\tilde{s}, \tilde{\tau})+\tilde{s} \partial_{\tilde{s}} \hat{w}(\tilde{s}, \tilde{\tau})+\left(\tilde{\tau}-\tau^{*}\right) \partial_{\tilde{\tau}} \hat{w}(\tilde{s}, \tilde{\tau})=0,
$$

which, together with (3.35), yields (1.10). Theorem 1.3 is established. $\square$

4. Proof of Theorem 1.2. In this last section we give the

Proof of Theorem 1.2. The ideas of the proof are already in the proof of Theorem 1.3. We only need to point out some changes.

Note that (1.2) holds if $u$ is not identically zero for the same reason, and we still have $u \in L_{l o c}^{\infty}\left(\mathbb{R}^{N}\right)$.

Lemma 1 still holds but we need to make the following changes.

Proof of Lemma 1. Follow the proof of Lemma 1 until (2.3). To prove (2.3), we note that the equation of $u_{\lambda}$ becomes

$$
-\Delta u_{\lambda}(\xi)=\left(\frac{\lambda}{|\xi|}\right)^{N+2-2 t-(N-2) p} \frac{u_{\lambda}^{p}(\xi)}{\left|\xi_{1}\right|^{t}} \leq \frac{u_{\lambda}^{p}(\xi)}{\left|\xi_{1}\right|^{t}}, \quad \text { in } \mathbb{R}^{N} \backslash B_{\lambda} .
$$

Thus, using (1.2) and the fact that $u \in L_{l o c}^{\infty}\left(\mathbb{R}^{N}\right)$,

$$
-\Delta\left(u(\xi)-u_{\lambda}(\xi)\right) \geq \frac{O(1)}{\left|\xi_{1}\right|^{t}}\left(u(\xi)-u_{\lambda}(\xi)\right), \quad \text { in } \mathbb{R}^{N} \backslash B_{\lambda} .
$$

Multiplying both sides of the above by $\left(u-u_{\lambda}\right)_{-}$and integrating by parts, we find, following (2.5) with obvious modification, that

$$
\begin{aligned}
\int_{B_{\lambda_{2} \backslash B_{\lambda}}}\left|\nabla\left(u-u_{\lambda}\right)_{-}\right|^{2} & \leq C \int_{B_{\lambda_{2} \backslash B_{\lambda}}} \frac{\left|\left(u-u_{\lambda}\right)_{-}\right|^{2}}{\left|\xi_{1}\right|^{t}} \\
& \leq\left. C\left|B_{\left.\lambda_{2} \backslash B_{\lambda}\right|^{\mid--t}} \int_{B_{\lambda_{2}} \backslash B_{\lambda}}\right| \nabla\left(u-u_{\lambda}\right)_{-}\right|^{2} .
\end{aligned}
$$

We reach a contradiction by taking $\lambda_{2}$ small enough. In (2.5), we did not use the fact that $u \in L_{l o c}^{\infty}\left(\mathbb{R}^{N}\right)$.

Lemma 2 and Lemma 3 can be put together as

Lemma 11. $\bar{\lambda}(x)=\infty$ for all $x=(0, z)$ in $\{0\} \times \mathbb{R}^{N-k}$.

Proof of Lemma 11. Lemma 2 still holds, and the proof is essentially the same, since the differential inequality (4.36), instead of an equality, is really what is needed in the proof. Lemma 3 still holds and the proof is the same. Now, since $p<t^{*}(t)-1$, i.e. the exponent $N+2-2 t-(N-2) p$ in (4.36) is positive, there is no way to have $u \equiv u_{x, \bar{\lambda}(x)}$. Indeed that would lead to

$$
\begin{aligned}
\frac{u^{p}(\xi)}{\left|\xi_{1}\right|^{t}}= & -\Delta u(\xi)=-\Delta u_{x, \bar{\lambda}(x)}(\xi)=\left(\frac{\bar{\lambda}(x)}{|\xi-x|}\right)^{N+2-2 t-(N-2) p} \frac{u_{x, \bar{\lambda}(x)}^{p}(\xi)}{\left|\xi_{1}\right|^{t}} \\
& =\left(\frac{\bar{\lambda}(x)}{|\xi-x|}\right)^{N+2-2 t-(N-2) p} \frac{u^{p}(\xi)}{\left|\xi_{1}\right|^{t}}, \quad \forall \xi .
\end{aligned}
$$


This is impossible since $N+2-2 t-(N-2) p>0$. Therefore $\bar{\lambda}(x)=\infty$ for all $x$. Lemma 11 is proved.

As before, $\bar{\lambda}(x) \equiv \infty$ implies that $u(y, z)$ is independent of the $z$-variables. But, for the same reasons given in the proof of Lemma 9, equation (1.3) does not have any positive radially symmetric solutions in $D_{l o c}^{1,2}\left(\mathbb{R}^{N}\right)$. Therefore $u \equiv 0$. Theorem 1.2 is established.

Added to the proof: We have recently been informed, by the authors, of the following related works: [9] and [17].

Acknowledgment. Part of this work was completed while Li was visiting the Institute of Applied Mathematics, AMSS, Chinese Academy of Sciences. He thanks his colleagues and friends at the institute for their hospitality.

\section{REFERENCES}

[1] A. Alvino, V. Ferone and G. Trombetti, On the best constant in a Hardy-Sobolev inequality, Appl. Anal., 85 (2006), pp. 171-180.

[2] H. Berestycki and L. NirenberG, On the method of moving planes and the sliding method, Bol. Soc. Brasil. Mat. (N.S.), 22 (1991), pp. 1-37.

[3] G. BiAnchi, Non-existence of positive solutions to semilinear elliptic equations on $\mathbb{R}^{n}$ or $\mathbb{R}_{+}^{n}$ through the method of moving planes, Comm. Partial Differential Equations, 22 (1997), pp. 1671-1690.

[4] H. Brezis and M. Marcus, Hardy's inequalities revisited, Dedicated to Ennio De Giorgi, Ann. Scuola Norm. Sup. Pisa Cl. Sci., 25 (1997), pp. 217-237 (1998).

[5] H. Brezis, M. Marcus ANd I. Shafrir, Extremal functions for Hardy's inequality with weight, J. Funct. Anal., 171 (2000), pp. 177-191.

[6] L. Caffarelli, B. Gidas and J. Spruck, Asymptotic symmetry and local behavior of semilinear equations with critical Sobolev growth, Comm. Pure Appl. Math., 42 (1989), pp. 271297.

[7] L. Caffarelli, R. Kohn and L. Nirenberg, First order interpolation inequalities with weights, Compositio Math., 53 (1984), pp. 259-275.

[8] D. CAO AND P. Han, Solutions for semilinear elliptic equations with critical exponents and Hardy potential, J. Differential Equations, 205 (2004), pp. 521-537.

[9] D. Castorina, I. Fabbri, G. Mancini and K. Sandeep, Hardy-Sobolev extremals, hyperbotic symmetry and scalar curvature equation, preprint.

[10] S. Filippas, V. Maz'ya and A. Tertikas, Critical Hardy-Sobolev inequalities, J. Math. Pures Appl., 87 (2007), pp. 37-56.

[11] N. Ghoussoub And C. YuAn, Multiple solutions for quasi-linear PDEs involving the critical Sobolev and Hardy exponents, Trans. Amer. Math. Soc., 352 (2000), pp. 5703-5743.

[12] B. Gidas, W.M. Ni And L. Nirenberg, Symmetry of positive solutions of nonlinear elliptic equations in $R^{n}$, Mathematical analysis and applications, Part A, pp. 369-402, Adv. in Math. Suppl. Stud., 7a, Academic Press, New York-London, 1981.

[13] D. Gilbarg and N.S. Trudinger, Elliptic Partial Differential Equations of Second Order, Second edition. Grundlehren 224, Springer, Berlin, Heidelberg, New York and Tokyo, 1983.

[14] Y.Y. Li AND L. Zhang, Liouville-type theorems and Harnack-type inequalities for semilinear elliptic equations, J. D'Analyse Math., 90 (2003), pp. 27-87.

[15] Y.Y. Li AND M. Zhu, Uniqueness theorems through the method of moving spheres, Duke Math, 80 (1995), pp. 383-417.

[16] G. Mancini, I. Fabbri and K. Sandeep, Classification of solutions of a critical Hardy-Sobolev operator, J. Differential Equations, 224 (2006), pp. 258-276.

[17] G. Mancini And K. Sandeep, On a semilinear elliptic equation in $\mathbb{H}^{n}$, preprint.

[18] J. Serrin AND H. Zou, Classification of positive solutions of quasilinear elliptic equations, Topology Methods in Nonlinear Analysis, J. Juliusz Schauder Center, 3 (1994), pp. 1-26.

[19] N.S. TRUDINGER, Remarks concerning the conformal deformation of Riemannian structures on compact manifolds, Ann. Scuola Norm. Sup. Cl. Sci. (3), 22 (1968), pp. 265-274. 
D. CAO AND Y. LI 